unclear. We aimed to investigate the association between sarcopenia and anabolic resistance (AR) and insulin resistance (IR), and the role of physical activity in age, gender matched children with CD.

Methods 18 fasted, male and female CD (on thiopurines \pm anti$\mathrm{TNF} \alpha)$ in deep remission (16 y, BMI=21) and 9 matched controls (Con) (16 y, BMI=21) drank a liquid meal (Ensure plus, $44 \mathrm{~g} \mathrm{CHO}, 14 \mathrm{~g}$ PRO, $11 \mathrm{~g}$ fat) at $\mathrm{t}=0$. Arterialised hand and venous forearm blood samples were collected concurrently and brachial artery blood flow measured at baseline and every 20 mins for 2 hours. Net balance of branched chain amino acids (BCAA) and glucose were derived, giving indices of skeletal muscle protein balance and IR. Subjects had a DEXA scan and handgrip dynamometer test on the day, and wore a pedometer and completed a food diary (for 3 days) to assess physical activity and food intake. Patient questionnaires (incl. IBD-fatigue) were completed.

Results Net BCAA balance across the whole 2 hours was lower in $C D$ vs Con $(-0.1 \pm 0.2 \mu \mathrm{mol} / \mathrm{min}$ vs $0.6 \pm 0.3 \mu \mathrm{mol} /$ $\min , p=0.05)$. Yet an initial response to feeding $(t=0$ to $\mathrm{t}=20)$ was exhibited by both $\mathrm{CD}(+1 \mu \mathrm{mol} / \mathrm{min})$ and Con $(+0.8 \mu \mathrm{mol} / \mathrm{min})$ but was only sustained post $40 \mathrm{mins}$ in Con. IBD-fatigue scores indicated CD had moderate fatigue (6), which had a moderate effect on daily activities (17). Handgrip dynamometer testing showed a trend towards greater fatigue in $\mathrm{CD}$ vs Con (+8\%pts) in the dominant arm $(\mathrm{p}=0.061)$. A trend towards lower total body lean mass in CD $(-15 \%$, $\mathrm{p}=0.084$ ) was found. No differences were detected in strength, physical activity, diet or IR. Thus despite not exhibiting AR (initial response to the meal) CD could not maintain a positive protein balance post feeding. This was associated with reduced muscle mass and function.

Conclusions The inability to sustain a positive protein balance postprandially could provide an explanation for the reduced muscle mass seen in $\mathrm{CD}$ patients in remission and be contributing to fatigue and poor muscle function. Pharmacological interventions to reduce protein breakdown and a high protein diet and /or exercise to improve anabolic response could be investigated as potential treatments.

\section{ADTU-02 V565, A NOVEL ORAL ANTI-TNF DOMAIN ANTIBODY, REDUCES COLONIC MUCOSAL INFLAMMATION IN PATIENTS WITH UC}

${ }^{1}$ Gareth Parkes*, ${ }^{2}$ Suhail Nurbhai, ${ }^{2}$ Mike West, ${ }^{2} \mathrm{~K}$ Ray, ${ }^{3} \mathrm{Tom}$ MacDonald ${ }^{3}$ Anna Vossenkaemper, ${ }^{2} S$ Crowe. ${ }^{1}$ Royal London Hospital, London, UK; ${ }^{2}$ VHSquared, Cambridge, UK; ${ }^{3}$ Centre for Immunobiology, Blizard Institute, QMUL, London, UK

\subsection{6/gutjnl-2018-BSGAbstracts.118}

Introduction Monoclonal antibodies to TNF transformed treatment options for patients with Inflammatory Bowel Disease (IBD). V565 is a novel oral anti-TNF domain antibody (Vorabody) engineered to be resistant to intestinal proteases. It is in development as a potential oral treatment for IBD. In vitro it suppressed phosphorylation of tyrosine kinases and signalling proteins and inhibited the release of inflammatory cytokines following culture with biopsies taken from patients with CD (Crowe et al. 18th International Congress of Mucosal Immunology, July 19-22 2017, Washington DC, USA). It was safe and well tolerated after high single and multiple doses in healthy volunteers and patient volunteers with $\mathrm{CD}$ and resulted in high concentrations of active drug in ileal fluid and faeces.
Aims \& Methods This open label study was designed to demonstrate that V565 enters GI mucosa and exerts a beneficial effect on inflammatory processes following oral dosing for 7 days to patients with Ulcerative Colitis. Patients with a Mayo score of 3-10 including an endoscopy score of $\geq 1$ had up to 7 days of oral dosing with $555 \mathrm{mg}$ tid V565. Sigmoidoscopy with biopsies was performed before and after the dosing period. The primary outcomes of interest were presence of V565 in the mucosa and reduction from baseline in phosphorylation of tyrosine kinases and signalling proteins. Detection of V565 was determined by immunohistochemistry. Phosphorylation was determined using PathScan RTK signalling arrays (Vossenkaemper et al 2014. Gastroenterology 147:172-83).

Results

Five patient volunteers were treated Due to visit scheduling, most received 6 days treatment. Presence of V565 was confirmed in the inflamed lamina propria and co-localised with CD14 + macrophages in post-treatment biopsies. Overall phosphorylation of the panel of kinases and signalling proteins was reduced by approximately $50 \%$ in four of the five patients. There were no treatment induced ADAs.

Conclusion V565, an oral anti-TNF domain antibody engineered to be resistant to intestinal proteases, was demonstrated bound to CD14 +macrophages in the lamina propria of UC patients and resulted in inhibition of mucosal inflammatory processes after 6-7 days oral dosing. The reduction of $50 \%$ in overall phosphorylation is similar to that seen in an earlier study of UC biopsy cultures with infliximab at a concentration of $67 \mathrm{nM}(10 \mu \mathrm{g} / \mathrm{ml})$, a serum concentration associated with mucosal healing (Ungar et al, Clin Gastroenterol Hepatol. 2016 Apr;14(4):550-557). These results provide encouragement that oral dosing with V565 will be a beneficial oral treatment option for patients with IBD.

\section{ADTU-03 IMPACT OF IMPROVED ACCESS TO BIOLOGIC THERAPIES AND PHYSICIAN ENGAGEMENT ON EXCESS STEROID EXPOSURE}

Gareth Parkes*, Christian Selinger, Ash Bassi, Melissa Smith, Jimmi Lindi, Helen Ludlow, Pritash Patel, Santosh Salunke, Veronica Hall, Becky George, John Saunders, Mark Adamson, Fiona Donovan, loanna Parisi, Lynn Gray, Aileen Fraser, Richard Pollock, Glyn Scott, Tim Raine. IBD Steroid Assessment Tool Group, Various, UK

\subsection{6/gutjnl-2018-BSGAbstracts. 119}

Background Steroid free remission is an important goal of IBD therapy. The aim of this study was to evaluate temporal changes in steroid prescribing in UK IBD outpatients in the context of major changes in UK prescribing guidelines and physician participation in audit and tailored service changes.

Methods Steroid use over the previous 12 months was recorded for unselected outpatient attenders against a definition of excess from ECCO guidelines. Data were collected from 7 centres that had completed a steroid assessment audit cycle in 2015, as well as from 12 new matched centres.

Results Data was collected for 2385 patients May-July 2017 and compared with 2015 data from 1176 patients. Overall disease distribution was $47.1 \% \mathrm{CD}, 49.6 \% \mathrm{UC}$ and $3.3 \%$ IBD-U, whilst $77.7 \%$ of patients were in clinical remission at the time of assessment. There was only a modest increase in patient exposure to anti-TNF from 2015 to 2017 : $30.6 \%$ to $37.2 \%$ in CD $(p=0.009)$ and $9.9 \%$ to $12.0 \%$ in UC $(p=N S)$. Anti-integrin usage increased from $0.8 \%$ to $3.3 \%$ in $\mathrm{CD}$ $(p=0.002)$ and from $1.6 \%$ to $2.4 \%$ in UC $(p=N S)$. For 
centres taking part in the 2015 audit, steroid exposure rates fell from $30 \%$ to $23.8 \%(p=0.003)$ and steroid excess from $13.7 \%$ to $11.5 \%$ ( $\mathrm{p}=\mathrm{NS}$ ). Steroid exposure and excess rates for sites that had not been part of the previous audit were significantly higher $(31.0 \%$ excess, $17.1 \%$ exposure, $\mathrm{p}=0.0001$ for both). There were no significant differences in important baseline characteristics of 2 groups of sites. Logistic regression analysis revealed independent predictors of reduced risk of steroid excess, after correction for disease severity. For CD these included treatment with anti-TNF therapy $(p=0.04)$, treatment in a centre with regular IBD multidisciplinary team (MDT) meetings $(p=0.01)$ and treatment in an original 2015 centre $(p=0.02)$. For UC treatment in a 2015 centre was also significant predictor of protection $(p=0.04)$ and treatment with thiopurine monotherapy a predictor of risk of excess $(p=0.01)$; usage of anti-TNF therapy in UC did not reach significance for protection from excess.

Conclusions Changes in biologic access in the UK have resulted in only modest changes in prescribing behaviour and have not yet impacted significantly on excess steroid exposure in UC, unlike in CD. Participation in an audit cycle of steroid usage was associated with a meaningful reduction in steroid excess. These data support the concept that steroid excess could be used as a key performance indicator in IBD and physicians should be engaged in this process.

\section{ADTU-04 FAECAL CALPROTECTIN IN PSC-IBD: A NOVEL MARKER OF CHOLANGITIS}

Polychronis Pavlidis*, Yasser El-Sherif, Ben Warner, Shraddha Gulati, Konstantinos Sarras, Ellie Alberts, Joe Segal, Tamir Rashid, Phil Harrison, John Devlin, Michael Heneghan, Alberto Fueyo, Gwion Emlyn, Hadil Abu Arqoub, Patrick Dubois, Deepak Joshi, Nick Powell, Bu' Hayee. King's Health Partners, London, UK

\subsection{6/gutjnl-2018-BSGAbstracts. 120}

Introduction Primary sclerosing cholangitis (PSC) is a chronic inflammatory condition of the bile ducts leading to fibrosis and end stage liver disease. A lack of robust non-invasive biomarkers has been hindering disease monitoring and development of optimal therapies. We have previously noted that the high levels of faecal calprotectin (fcal) seen in PSC-IBD patients belie the mild or quiescent intestinal inflammation. An unsupervised proteomics study identified biliary calprotectin as a potential biomarker. Here, we test the hypothesis that fcal is a marker of biliary injury in PSC.

Methods We analysed paired endoscopic activity data (UCEIS) and fcal results of patients with PSC-IBD $(n=20)$ or UC $(n=20)$ who underwent colitis surveillance in the context of a colitis surveillance pilot study. Relevant clinical data was recorded prospectively. Recruiting consecutive patients attending for ERCP $(n=6)$ allowed for the concomitant testing of biliary and faecal calprotectin.

Results As expected, fcal strongly correlated with severity of mucosal injury (UCEIS) in UC $[\mathrm{r}=0.82,95 \% \mathrm{CI}(0.58,0.92)$, $\mathrm{p}<0.0001]$. However, the correlation was weaker in PSC-IBD $[\mathrm{r}=0.59,95 \% \mathrm{CI}(0.19,0.82), \mathrm{p}=0.006]$. Moreover, in patients with PSC-IBD and quiescent colitis (UCEIS: 0-1) fcal concentration was significantly higher in comparison to UC patients with comparable endoscopic activity $[279 \mathrm{ug} / \mathrm{g}(10,1560)$ vs. $30(10,161), p=0.015)]$. A trend towards abnormal liver biochemistry was seen in those PSC-IBD with higher fcal [ALP: 250IU/L $(113,561)$ vs. $83(59,170), \mathrm{p}=0.06$, GGT: $351 \mathrm{U} / \mathrm{L}$
(117, 1014) vs. $51(29,153) \mathrm{p}=0.02$, AST: $53 \mathrm{U} / \mathrm{L}(26,85)$ vs. $37(22,43), \mathrm{p}=\mathrm{ns}$. UC patients with quiescent colitis and fcal $>150$ had a higher risk of colitis relapse in 12 months $[\mathrm{HR}=7.6,95 \% \mathrm{CI}(1.8,33.6)]$ in comparison to those with fcal $<150$. However, in patients with PSC-IBD and quiescent colitis a fcal $>150$ was associated instead with a higher risk of cholangitis associated complications (need for antibiotics or stent insertion), $\mathrm{HR}=6.5,95 \% \mathrm{CI}(1.3,33.9)$. Strikingly, biliary calprotectin concentration showed a strong correlation with fcal concentration $(r=0.90, p=0.04)$. Interestingly, immunostaining of biliary brushings for calprotectin demonstrated positive staining in cholangiocytes as well as neutrophils and macrophages.

Conclusion In patients with PSC-IBD and quiescent colitis the identification of a raised fcal is likely to herald complications of inflammation in the bile ducts rather than the colon. In this setting, fcal may be a valuable prognostic biomarker of cholangitis. Additionally, our data suggest that in PSC, the source of raised fcal may also be the damaged biliary epithelium.

\section{ADTU-05 GUT MICROBIAL COMPOSITION IN THE MIGRANT SOUTH-ASIAN IBD POPULATION IN UK}

${ }^{1,2}$ Mohammed Quraishi* ${ }^{3}$ Animesh Acharjee, ${ }^{2,3}$ Neeraj Bhala, ${ }^{2}$ Shrikanth Pathmakanthan,
${ }^{2}$ Rachel Cooney, ${ }^{2,3}$ Subrata Ghosh, ${ }^{3}$ Georgios Gkoutos, ${ }^{1,2}$ Andrew Beggs,
${ }^{4}$ Amanda Rossiter* ${ }^{1,2,3}$ Tariq Iqbal ${ }^{*} .{ }^{1}$ Institute of Cancer and Genomic Sciences, University
of Birmingham, Birmingham, UK; ${ }^{2}$ University Hospital Birmingham, Birmingham, UK;
${ }^{3}$ Institute of Translational Medicine, Birmingham, UK; ${ }^{4}$ Institute of Microbiology and
Infection, University of Birmingham, Birmingham, UK

\subsection{6/gutjnl-2018-BSGAbstracts. 121}

Introduction Epidemiological studies have highlighted that the South Asian migrant population in UK have a comparable risk of developing IBD but with a more aggressive phenotype than the white Caucasian population. It remains unclear if this is due to environmental/lifestyle factors or differences in host genetics. The human gut microbiota is impacted by health status and diet and therefore represents a potentially adaptive phenotype that is influenced by the environment. As the gut microbiota has been shown to be different in the native South Asian population compared to those in developed countries, we aimed to investigate if there were ethnic differences in the microbiota in IBD patients.

Methods Stool samples were collected from South Asian $(n=20)$ and Caucasian patients $(n=46)$ with IBD attending outpatient clinics at University Hospital Birmingham along with healthy controls $(n=17)$. DNA was extracted and the V4 hyper-variable region of the $16 \mathrm{~S}$ rRNA gene amplified and sequenced. Analysis was performed on the QIIME pipeline using the GreenGenes database. Diversity analysis was corrected for false discovery rates.

Result Patients with IBD had a significantly different gut microbial composition in comparison to healthy controls as expected $(p=0.01)$. Gut microbial diversity was reduced in IBD $(p=0.001)$. A significant decrease in Firmicutes phylum was observed in patients with IBD in comparison to healthy controls which was primarily due a reduced abundance of communities from genus Faecalibacterium prannitizii, Lachnospiracae, Ruminococcus and Blautia $(\mathrm{p}<0.002)$. Within the IBD cohort, the alpha or beta diversity of gut microbiota was very similar for South Asians and Caucasian patients. No significant differences were seen at any taxonomic levels. 